\title{
Artificial Neural Network Modeling of Properties of Crude Fractions with its TBP and Source of Origin and Time
}

\author{
S. L. Pandharipande \\ Associate Professor, \\ Chemical Engg. Dept. \\ Laxminarayan Institute of \\ Technology, Nagpur
}

\author{
Aditaya Akheramka \\ B. Tech Petroleum \\ Refining \& Petrochemical \\ Technology \\ Laxminarayan Institute of \\ Technology, Nagpur
}

\author{
Ankit Singh \\ M. Tech Chemical \\ Engineering \\ Laxminarayan Institute \\ of Technology, Nagpur
}

\author{
Anish Shah \\ M. Tech Chemical \\ Engineering \\ Laxminarayan Institute \\ of Technology, Nagpur
}

\begin{abstract}
The objective of present work is to inculcate the effect of the sources of crude as one of the input parameters along with volume fraction, sulfur content \& specific gravity of the crude on the estimation of mean average boiling point, molecular weights by developing ANN model. It is further extended to include the effect of time element on these properties of crude for one particular source of crude. Eleven sources of crude have been selected for first part of the work \& for the one particular source twenty samples at different time elements have been used. The developed ANN models are observed to be with the average accuracy of prediction within $\pm 1 \%$ .Based on the outcome of this demonstrative work, it can be concluded that ANN has a great potential in addressing to the estimation problems related to crude properties. The novel feature of the present work is incorporation of the origin of crude \& time elements along with the other properties in the ANN model developed for the prediction of important parameters like mean average boiling point \& molecular weight. It is sincerely felt that the methodology adopted in the present work be extended to more comprehensive data sets.
\end{abstract}

\section{Keywords}

Artificial neural network modeling, crude source, Petroleum fraction physical properties, TBP, time element

\section{INTRODUCTION}

Crude petroleum can be considered as the most important material, essential for sustaining the life cycle of the modern society. Its production begins from the exploration \& drilling of rigs in oil reserves, transportation through an extensive pipeline network to the refineries and separation, mostly on the basis of difference in boiling points. It is then further processed to give a large number of intermediates and consumer products, from petrol \& kerosene to asphalt and chemical reagents which find their applications in allied industries plastics, paints, fertilizers, insecticides, medicine and elsewhere.

The composition of crude petroleum varies widely from source to source and with time, having hydrogen and carbon as the major elements, whereas sulfur, nitrogen, oxygen and metals are present in a relatively smaller quantity. Petroleum crude is thus a mixture of hundreds of hydrocarbons ranging in size from the smallest, methane, with only one carbon atom, to large compounds containing 300 and more carbon atoms.

The crude oil assay is a compilation of the basic properties of the crude established through several laboratory and pilot plant tests. The variations in the hydrocarbon composition of the crude oil can be related to the physical properties of the crude and variations in true boiling point data, sulfur content and API gravity for the entire range of crude oil in increasing molecular weights, are contained as data in a typical crude assay. The properties reported in a crude assay are then, by means of empirical correlations, used directly to estimate other properties, whose laboratory estimation is difficult.

True boiling point data is determined by ASTM D-285 method which is performed in a fifteen theoretical plate fractionating column with a reflux ratio of five. It indicates the quantities of the various boiling point fractions present in the crude. Similarly, the data for the gravity and sulfur content of the crude samples are indicative of the suitability of the crude for a particular refinery having a definite infrastructure of process equipment.

Molecular weight determination of a fraction is tedious and difficult and is usually estimated using standard correlations involving parameters like normal boiling point and the specific gravity of the fractions. Accurately describing the molecular weight is required in energy and mass balance equations and the designing of the process equipment in the refinery.

There have been several correlations established in estimation of thermo physical properties of the crude fractions based on the crude TBP and the specific gravity data. Efforts have been made to use this data in selection and design of the process equipment in the refining and separation. However, little efforts have been made in correlating these characteristics of 
the crude and its fractions to the source in single mathematical model.

The present work aims to develop models inculcating the effect of the source of crude \& time element as independent parameters along with the other properties of the crude like volume fraction, sulfur content \& specific gravity of the crude. These are used as independent parameters in estimation of dependent parameters like mean average boiling point of the fraction and molecular weight of the fraction. The correlation technique adopted in the present work is Artificial Neural Networks.

\subsection{Artificial neural network}

Artificial Neural Network (ANN) is becoming a popular modeling tool for processes where non linear multivariable relationships are involved. ANN modeling is also called as black box modeling \& its working principle is loosely modeled on the biological neural network. It is composed of a large number of data processing elements called as nodes or neurons arranged in layers \& interconnected with each other to develop a correlation [1].

Multi-Layer Perceptron (MLP) is the most common type of ANN employed in modeling of chemical processes. It is feed forward neural network \& consists of input \& output layers apart from at least one hidden layer in between them. The number of nodes in input \& output layers is decided by the number of independent $\&$ dependent parameters that define the process. The number of hidden layers \& the number of nodes in each hidden layer are dependent upon the complexity of the model, the number of input $\&$ output nodes $\&$ the data sets involved. Nodes in successive layers are connected to each other by means of constants called as weights. The outputs from the input layer are fed to hidden layer units, which in turn, feed their outputs to the next hidden layer nodes. The training of MLP is usually carried by error back propagation algorithm suggested by Rumelhart [2]. There are number of applications of ANN, that include, , modeling of distillation column [3], detergent formulation [4], modeling of unsteady heat conduction in semi infinite solid [5], an approach to fault diagnosis in chemical processes [6], fault diagnosis in complex chemical plants [7], incipient fault diagnosis of chemical process [8], leak detection in liquefied gas pipeline $[9,10]$, prediction of mass transfer coefficient in downflow jet loop reactor [11], modeling of packed column [12], modeling steam tables [13] and Gurney Lurie charts [14] are also reported.

Efforts have also been made by researchers to use the intelligence tools, and in particular Artificial Neural Network, to develop predictive models for the determination of crude related properties. These include, ANN as a predictive tool for the determination of crude PVT properties for the data from the Middle East crude oil [15], using Artificial Neural Networks to develop new PVT correlations for Saudi crude oil [16], Artificial Neural Network models for predicting PVT properties of oil field brines [17] and evaluation of crude oil property using intelligence tools; fuzzy model approach [18].

\section{METHODOLOGY}

In the present work, the data related to different crude sources $\&$ for varying time elements has been used from the data available \& reported [19]. Two consolidated sets comprising data from eleven oil locations $\&$ twenty time elements for one particular source are compiled and parameters like mean average boiling point of the fraction \& molecular weight of the fraction have been calculated. The correlations used in the estimation of these parameters are listed in equations 1 \& 2 [20].

$$
\text { 1. }(\mathrm{MeABP})_{\mathrm{i}}=\frac{(\mathrm{NBP}) \mathrm{i}+(\mathrm{NBP}) \mathrm{i}-1}{2}
$$

Where MeABP $=$ Mean Average Boiling Point for a fraction, ${ }^{\circ} \mathrm{F}$

NBP $=$ Normal Boiling Point of $i^{\text {th }}$ volume fraction distilled off, ${ }^{\circ} \mathrm{F}$

2. $\mathrm{MW}=42.965\left[\exp \left(2.097 \times 10^{-4} \quad \mathrm{~T}_{\mathrm{b}} 7.78712 \mathrm{SG}\right.\right.$ $\left.\left.+2.08476 \times 10^{-3} \mathrm{~T}_{\mathrm{b}} \mathrm{SG}\right)\right] \mathrm{T}_{\mathrm{b}}{ }^{1.26007} \mathrm{SG}^{4.98308}$

Where MW = Molecular weight of the fraction,

$\mathrm{T}_{\mathrm{b}}=$ Mean average boiling point of the fraction, Kelvin

$\mathrm{SG}=$ Specific gravity, $60^{\circ} \mathrm{F} / 60^{\circ} \mathrm{F}$ 


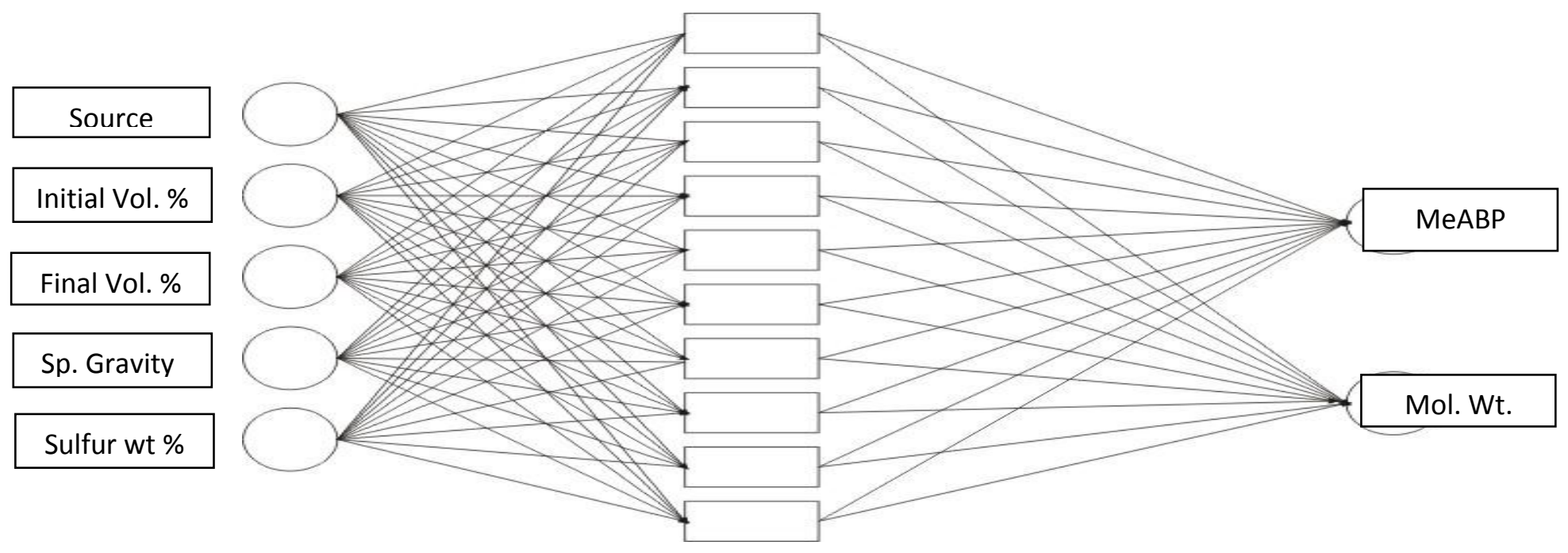

Figure 01: A typical architecture of the topology of the ANN model 1

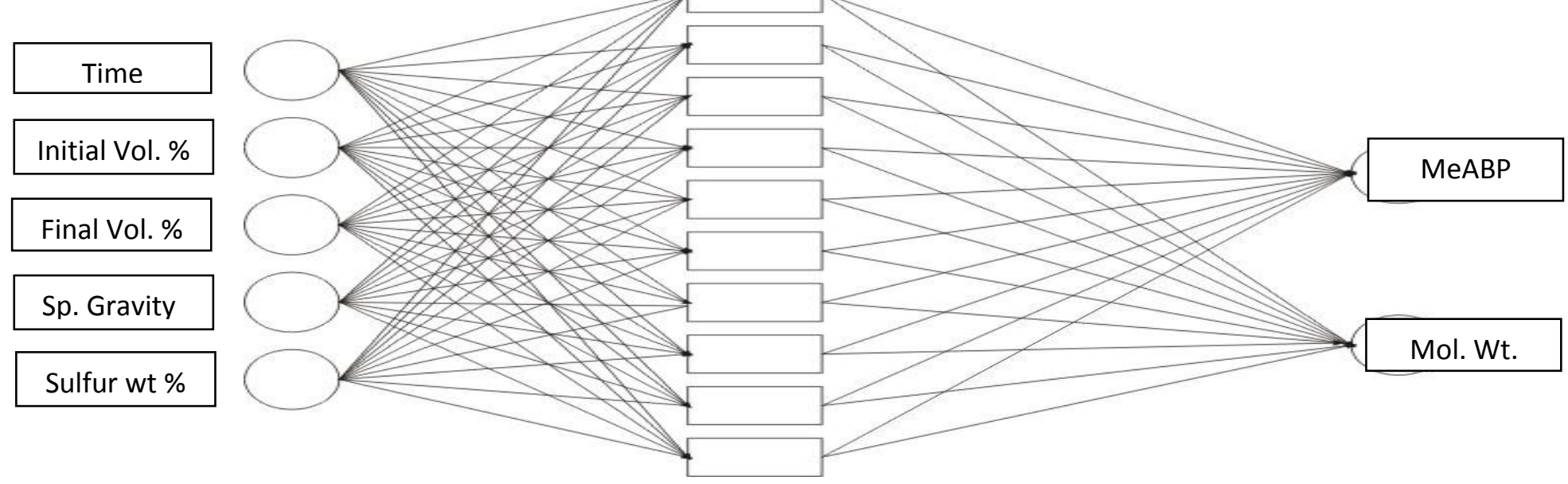

Figure 02: A typical architecture of the topology of the ANN model 2

Two separate ANN models are developed using eLITe $\mathrm{ANN}^{\odot}$ [21], one each for the two parts of the present work. The architectures of the topology for ANN models $1 \& 2$ are depicted in the Fig $1 \& 2$.

The input parameters include coded values for the 11 different sources of the crude, initial volume percent of the fraction, final volume percent of the fraction, specific gravity of the crude and the sulfur weight percent of the crude for ANN model 1. The various sources of the crude included in this model are Statoil Cheecham Blend, Suncor Synthetic H, Surmont Heavy Blend, Boundary Lake BDY-915, Boundary Lake BDY-012, Peace River Sour, Lloyd Kerrobert, Bow River North, Koch Alberta, Medium Gibson Sour, Mixed Sour Blend.
Similarly for ANN model 2, the input parameters included are the coded values for the different cumulative time elements for BC Light crude, initial volume percent of the fraction, final volume percent of the fraction, specific gravity of the crude and the sulfur weight percent of the crude.

The special feature of the present work is the incorporation of coded value for source of the crude \& time elements which are otherwise difficult to be incorporated in the conventional estimation methods. The output parameters are the mean average boiling point of the fraction and the average molecular weight of the fraction.

\section{RESULTS}

The details of the topology of the ANN models 1 \& 2 developed are shown in table no. 01 . 
Table 01: Details of the topology of the two ANN models developed

\begin{tabular}{|c|c|c|c|c|c|c|c|}
\hline \multirow[t]{2}{*}{ Model } & \multicolumn{5}{|c|}{ No. of neurons } & \multirow[t]{2}{*}{ Data points } & \multirow[t]{2}{*}{ RMSE } \\
\hline & $\begin{array}{l}\text { Input } \\
\text { layer }\end{array}$ & $\begin{array}{c}\mathbf{1}^{\text {st }} \\
\text { Hidden } \\
\text { layer }\end{array}$ & $\begin{array}{c}2^{\text {nd }} \\
\text { Hidden } \\
\text { layer }\end{array}$ & $\begin{array}{c}3^{\text {rd }} \\
\text { Hidden } \\
\text { layer }\end{array}$ & $\begin{array}{c}\text { Output } \\
\text { layer }\end{array}$ & & \\
\hline ANN Model 1 & 5 & $\mathbf{0}$ & 10 & 10 & 2 & 204 & 0.00322 \\
\hline ANN Model 2 & 5 & $\mathbf{0}$ & 10 & 10 & 2 & 397 & 0.00488 \\
\hline \multicolumn{8}{|c|}{ Number of Iterations $=\mathbf{5 0 , 0 0 0}$} \\
\hline
\end{tabular}

Total data set of $204 \& 397$ points have been used in developing ANN model $1 \& 2$ respectively using elite-ANN ${ }^{\odot}$. Comparison of actual and predicted values of the output parameters, mean average boiling point of the fraction and molecular weight of the fraction, for ANN models $1 \& 2$ is shown in figures 3 to 6 . As can been seen from these comparisons the predicted values are very close to the actual values for all the output parameters \& indicative of the successful development of models in predicting crude properties incorporating origin of crude \& time elements.

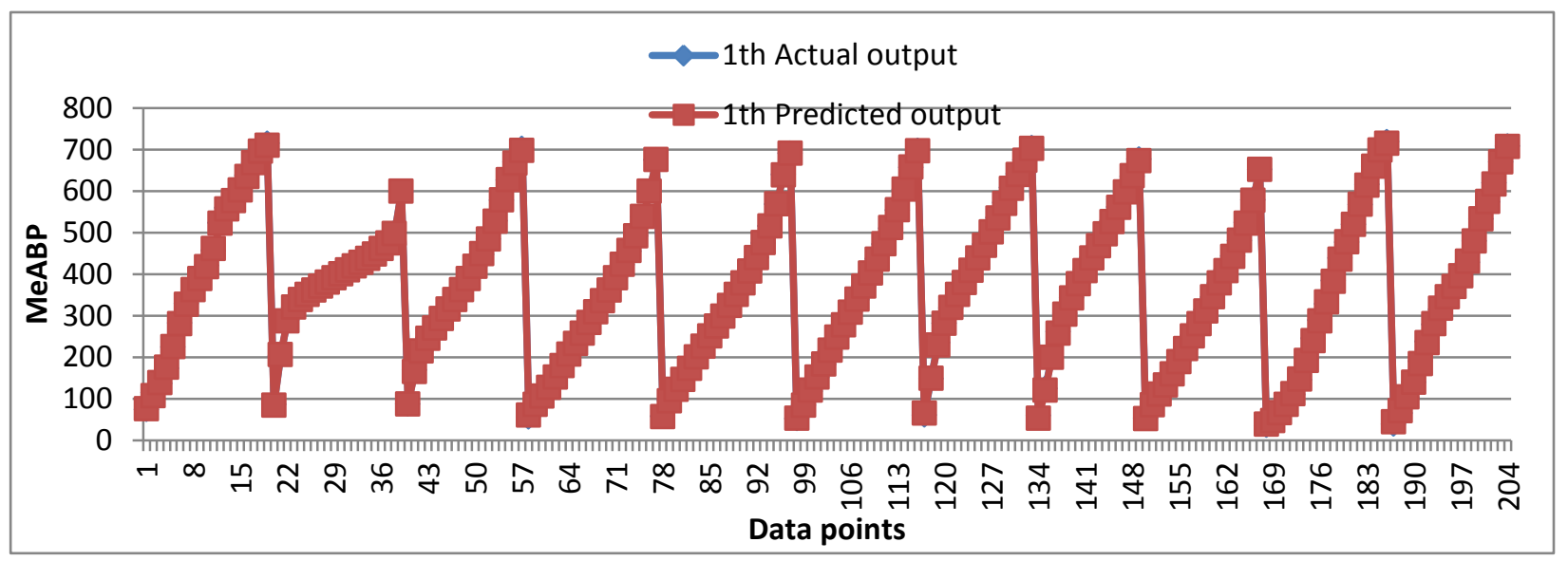

Figure 3: Comparison of mean average boiling point of actual and predicted values for ANN model 1

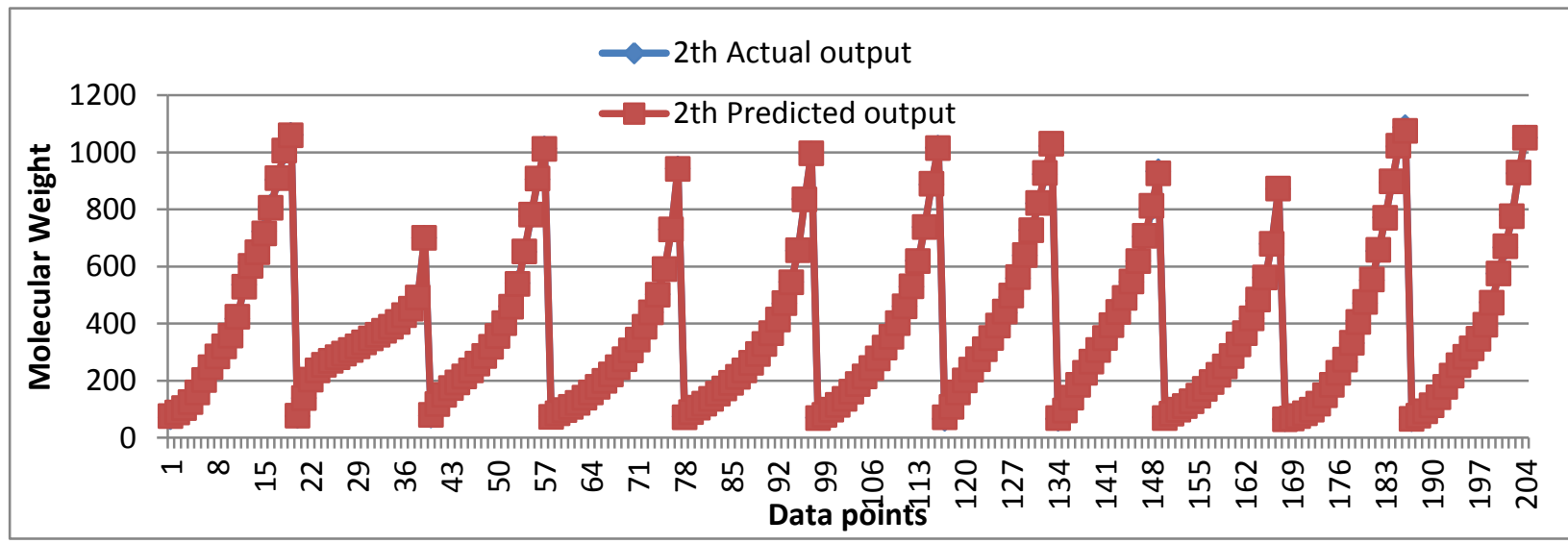

Figure 4: Comparison of molecular weight of actual and predicted values for ANN model 1 


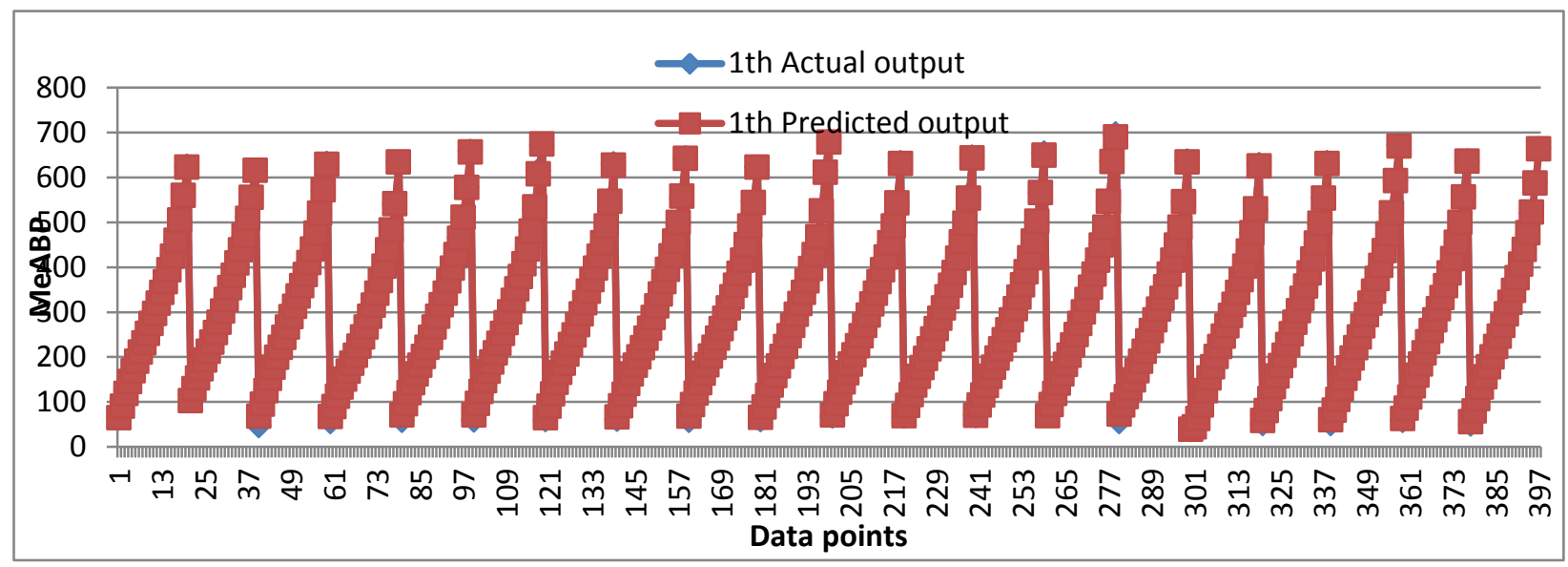

Figure 5: Comparison of mean average boiling point of actual and predicted values for ANN model 2

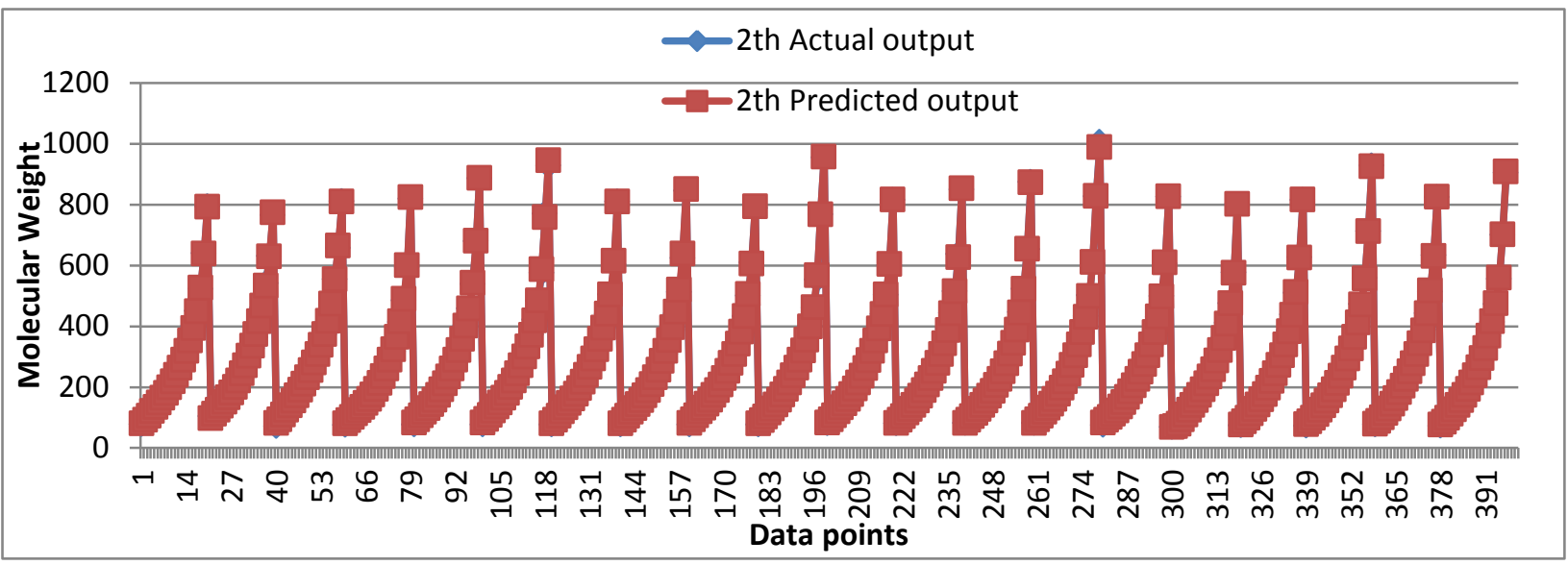

Figure 6: Comparison of molecular weight of actual and predicted values for ANN model 2

\section{CONCLUSION}

Proper \& adequate supply of petroleum crude is the lifeline of modern world and the limited stocks have made it a centre stage theme for the researchers, designers, planners \& explorers. The estimations on the quality of the crude as a function of its source are one of the important areas of interest and very little has been reported in the literature. The theme becomes more complex as the quality of crude from the well is also function of the time element. The objective of present work is to explore the possibility of development of ANN models for estimation of crude properties like mean average boiling point $\&$ molecular weight of the fraction as a function of coded value for origin of crude \& cumulative time elements for a particular crude source in addition to the other input parameters like volume fraction, sulfur content \& specific gravity of the crude. The novel feature of the present work is incorporation of eleven sources of crude in a single ANN model 1 \& incorporation of cumulative time elements in ANN model 2.Very little has been reported in the literature in this regard. It is observed that the average value of accuracy of prediction is within $\pm 1 \%$ which is highly acceptable. The present work is demonstrative \& based on the outcome it can be concluded that ANN has great potential in addressing to the estimation problems related to crude incorporating the source \& cumulative time elements along with the other properties for the prediction of important parameters like molecular weight among others \& should be extended to more comprehensive data sets. This would save on the time and energy and increase the accuracy of the estimations.

\section{ACKNOWLEDGEMENT}

Authors are thankful to Director, LIT, Nagpur for the facilities and encouragement provided. Authors also wish to express the sincere gratitude towards the officials of Crude Quality Inc. \#200, 17850105 Avenue, Edmonton, Alberta Canada for giving the permission to use the crude source data for the present work.

\section{REFERENCES}

[1] Anderson J.A, An Introduction to Neural Networks Prentice-Hall of India, Pvt Ltd New Delhi, (1999).

[2] Rumelhart D E \& McClleland Back Propagation Training Algorithm Processing, M.I.T Press, Cambridge Massachusetts, (1986).

[3] Baratti R, Vacca G \& Servida A, Hydrocarbon, (1995) 35.

[4] Pandharipande S L, Agarwal R S, Gogte B B \& Badhe Y P, Chem Eng World 38 (5) (2003) 78-80 
[5] Pandharipande S L \& Badhe Y P, Chem Eng World 38 (8) (2003) 82.

[6] Fan J Y, Nikolau M \& White R E, AIChE, 39 (1) (1993) 82.

[7] Hoskins J C, Kaliyur K M \& Himmelblau D M, AIChE, 37(1) (1991) 137.

[8] Watanabe K, Abe M, Kubota M \& Himmelblau D M, AIChE, 35 (11) (1989) 1803.

[9] Belsito S \& Banerjee S AIChE 44 (12), (1998), 2675.

[10] Pandharipande S L \& Badhe Y P, Chem Eng World 38(6) (2003) 70

[11] Pandharipande S L \& Badhe Y P IIChe, 45(4) (2003) 256.

[12] Pandharipande S L \& Mandavgane S A, Indian J Chem Technol, 11 (6) (2004) (820)

[13] Pandharipande S L, Bhaise A \& Poharkar A, Chem Eng world, 39 (1) (2004) 50
[14] Pandharipande S L \& Badhe Y P, J Inst Eng, 84 (3) (2004) 65.

[15] Gharbi R.B. , Elsharkawy A.M., Middle East Oil Show \& Conference, $15-18^{\text {th }}$ March 1997,Bahrain

[16] Marhoun M.A., Osman P.A.,Abu Dhabi international Petroleum Exhibition \& Conference $13-16^{\text {th }}$ October 2002, Abu dhabi,UA

[17] Osman P.A., E., Marhoun M.A.,SPE Middle East Oil \& Gas Show \& Conference, 12-15 ${ }^{\text {th }}$ March 2005,Kingdom of Bahrain.

[18] Reza Abedini, Morteza Esfandyari,Amir Nezhadmoghadam,Hooman Adib,Chem Engg Research Bulletin, 15(2011)30-33.

[19] Crude source data, www.crudemonitor.ca

[20] Fahim M.A., Alsahhaf T.A., Elkilani M.A., Fundamentals of Petroleum Refining, Elsevier 2010.

[21] Pandharipande S L \& Badhe Y P, elite-ANN ${ }^{\odot}$, ROC No SW-1471/2004. 